\title{
Estado nutricional antropométrico e qualidade de vida em escolares com asma
}

\author{
Anthropometric nutritional status and quality of life in students with asthma
}

\author{
Aline do Amaral Mendes', Márcio Júnior Strassburger¹, Lígia Beatriz Bento Franz¹, Maristela Borin Busnello', \\ Iara Denise Endruweit Battisti ${ }^{2}$, Simone Zeni Strassburger ${ }^{1} \bowtie$ \\ ${ }^{1}$ Departamento de Ciências da Vida da Universidade Regional do Noroeste do Estado do Rio Grande do Sul (UNIJUI). Ijuí, RS. \\ 2 Universidade Federal da Fronteira Sul (UFFS). Cerro Largo, RS.
}

\section{RESUMO}

Objetivos: Investigar a relação entre estado nutricional antropométrico e qualidade de vida em escolares com asma.

Métodos: Estudo do tipo caso-controle com escolares de terceiro a oitavo ano do ensino fundamental no município de Ijuí, Rio Grande do Sul. A primeira etapa constituiu-se de um estudo transversal, sendo enviado aos domicílios dos estudantes um questionário de triagem, para ser preenchido pelos responsáveis, visando identificar os escolares com asma, os quais foram alocados para o grupo de casos (respostas positivas para tosse ou sibilância sem resfriado, uso de qualquer medicação para asma nos últimos 12 meses e/ou diagnóstico médico de asma). Para o grupo controle foram selecionados escolares pareados por sexo e idade cujas respostas foram negativas para asma, na proporção de dois controles para cada caso. Os escolares receberam visita domiciliar de pesquisadores treinados, que apresentaram aos participantes e seus pais/responsáveis um questionário sobre qualidade de vida relacionada à saúde. A avaliação do estado antropométrico foi realizada nas escolas, pelos pesquisadores.

Resultados: Foram investigados 1.691 escolares na primeira etapa do estudo, sendo 184 (10,8\%) classificados com asma. Destes, 486 escolares responderam ao questionário de qualidade de vida: 122 com asma e 364 controles. A avaliação antropométrica foi realizada em 414 escolares (106 casos e 308 controles). Crianças eutróficas totalizaram 237 (57,2\%), com baixo peso 8 (1,9\%), com sobrepeso 90 (21,7\%) e com obesidade 79 (19,1\%). Encontrou-se sobrepeso/obesidade em $49(46,2 \%)$ estudantes do grupo com asma e em 120 (38,9\%) do grupo controle $(\mathrm{p}<0,001)$. Comparando a qualidade de vida entre os grupos, no grupo com asma foram observados escores menores no domínio bem-estar físico $(p<0,001)$ e no escore total de qualidade de vida $(\mathrm{p}=0,003)$. Nos escolares com asma e sobrepeso/obesidade, a média do escore no domínio bem-estar físico apresentou-se menor quando comparado ao grupo sem asma e eutrófico ( $\mathrm{p}=0,010)$.

Conclusões: Quando comparados escolares entre sete e 18 anos com e sem história de asma, a asma associou-se com pior qualidade de vida. Considerando-se sobrepeso e obesidade como comorbidades, a qualidade de vida foi pior para esse grupo de escolares no domínio bem-estar físico.

DESCRITORES: asma; antropometria; saúde do adolescente; qualidade de vida.

\section{ABSTRACT}

Aims: To investigate the relationship between anthropometric status and quality of life in children with asthma.

Methods: Case-control study, including students from third to eighth grade of elementary school at the city of Ijuí, Rio Grande do Sul, Brazil. The first phase of the study consisted of a cross-sectional study, when a questionnaire of screening was sent to the students' homes to be completed by the parents or guardians, aiming to identify children with asthma, which were allocated to the group of cases (positive responses to cough, wheezing without colds, use of any medication for asthma in the last 12 months and/or medical diagnosis of asthma). For the control group, students matched by sex and age whose responses were negative for asthma were selected, at the rate of two controls for each case. The children received home visits from trained researchers, who presented to the participants and their parents/guardians a questionnaire about health related quality of life. Evaluation of anthropometric status was carried out at the schools by the researchers.

Results: In the first phase od the study 1,691 students were investigated, and $184(10.8 \%)$ were classified as having asthma. Of these, 486 students responded to the questionnaire of quality of life: 122 with asthma and 364 controls. Anthropometric assessment was performed in 414 children (106 cases and 308 controls). Eutrophic children totaled 237 (57.2\%), low weight 8 (1.9\%), overweight 90 (21.7\%) and obese $79(19.1 \%)$. Overweight/obesity was observed in $49(46.2 \%)$ students of the group with asthma and in 120 (38.9\%) in the control group $(\mathrm{p}<0.001)$. When comparing the quality of life between the groups, lower scores were observed among the asthma group in the physical well-being domain $(\mathrm{p}<0.001)$ and in the total score of quality of life $(\mathrm{p}=0.003)$. In children with asthma plus overweight/obesity, the mean score on the physical well-being domain was lower when compared to the group without asthma and eutrophic $(\mathrm{p}=0.010)$.

Conclusions: When comparing students between seven and 18 years with or without history of asthma, asthma was associated with poor quality of life. Considering overweight and obesity as comorbidities, quality of life was worse for this group of students in the physical wellbeing domain.

KEY WORDS: asthma; anthropometry; adolescent health; quality of life. 
Abreviaturas: ISAAC, International Study of Asthma and Allergies in Childhood; IMC, índice de massa corporal; $\mathrm{QV}$, qualidade de vida.

\section{INTRODUÇÃO}

Asma é uma doença inflamatória crônica das vias aéreas inferiores associada à hiper-responsividade brônquica [1]. É uma das mais comuns condições crônicas que afetam tanto crianças quanto adultos, sendo um problema mundial de saúde, acometendo cerca de 300 milhões de indivíduos, com elevada prevalência na infância. O projeto International Study of Asthma and Allergies in Childhood (ISAAC), em sua fase III, incluiu dados de várias cidades brasileiras entre os anos de 2001 e 2005, identificando prevalência de asma entre $21-22 \%$ em adolescentes de 13 a 14 anos [2].

A asma apresenta características etiológicas multifatoriais; o aparecimento dos sintomas está associado tanto a fatores genéticos quanto ambientais. Algumas comorbidades relacionadas aos fatores etiológicos podem se apresentar nas pessoas com asma, incluindo doenças alérgicas como rinite, rinoconjuntivite e dermatite atópica. Além disso, como se trata de uma doença crônica, comorbidades não relacionadas ou com relação indireta com a asma podem ocorrer, como a obesidade [3].

O sedentarismo, considerado um fator que contribui para a atual e preocupante "epidemia de obesidade infantil", junto com o aumento da prevalência de asma na infância, tem sido um assunto de interesse da ciência, com crescentes evidências de que a obesidade pode ser considerada um fator de risco para a asma, ligando assim as duas epidemias [4,5]. Além de a obesidade ser considerada um fator de risco para a asma, parece estar associada ao pior controle da doença, com frequente exacerbação dos sintomas e redução da tolerância ao exercício físico [6]. Da mesma forma, o aumento de peso também pode causar uma desvantagem na mecânica pulmonar, levando à diminuição da capacidade funcional, volume pulmonar e do diâmetro das vias aéreas [7].

As repercussões da asma e da obesidade atingem não somente o paciente, mas todo o universo familiar, podendo trazer problemas complexos e implicações a longo prazo, interferindo assim na QV desses indivíduos [8]. Embora se conheçam os efeitos negativos das duas doenças sobre a $\mathrm{QV}$, a associação de ambas é um assunto recente [9]. Estudo realizado por Gent et al. [10] com crianças entre sete e 10 anos, avaliou o impacto da obesidade e da asma na $\mathrm{QV}$, verificando que crianças portadoras de asma com índice de massa corporal (IMC) elevado apresentaram menores escores de QV quando comparadas às crianças sem essa condição [10]. Em outro estudo, realizado com 337 crianças e adolescentes, foi encontrada uma redução significativa da QV nos adolescentes com asma e sobrepeso/obesidade quando comparados aos indivíduos sem asma e eutróficos [11].

Contudo, a literatura acerca das relações entre asma, sobrepeso/obesidade e QV ainda é escassa. Dessa forma, o objetivo deste estudo foi investigar a relação entre o estado nutricional antropométrico e a QV em escolares com asma.

\section{MÉTODOS}

Foi realizado um estudo do tipo caso-controle, com escolares entre a terceira e a oitava séries do ensino fundamental, matriculados em quatro escolas municipais e quatro escolas estaduais do município de Ijuí, no interior do estado do Rio Grande do Sul. Essas escolas foram selecionadas por terem o maior número de alunos matriculados entre as escolas do município e por sua localização geográfica (abrangendo as regiões norte, sul, leste e oeste), procurando com isso representar os escolares das diversas regiões do município. O protocolo de pesquisa foi aprovado pelo Comitê de Ética em Pesquisa da UNIJUÍ, pelo parecer 246.061/2013.

A primeira etapa (etapa I) da pesquisa consistiu em um estudo transversal, em que foi realizada uma triagem, utilizando um questionário para classificação dos escolares com asma, na qual foram incluídos todos os escolares matriculados nas séries definidas para este estudo. Foi utilizado um breve questionário com parte das perguntas referentes ao diagnóstico epidemiológico de asma integrantes do ISAAC [12]. A entrega do questionário ocorreu em sala de aula, sendo que na mesma oportunidade foi realizada a leitura das perguntas, explicando o seu significado aos estudantes, sendo estes orientados a entregar o questionário e um Termo de Consentimento Livre e Esclarecido (TCLE) para seus respectivos responsáveis. Após preenchimento do questionário e assinatura do TCLE pelos responsáveis, os questionários deveriam voltar para a escola. Pela análise das respostas ao questionário de triagem, foi possível definir os escolares que apresentavam asma ativa, definida como tosse ou sibilância sem resfriado nos últimos 12 meses, uso de qualquer medicação para asma nos últimos 12 meses 
e/ou diagnóstico de asma feito por um médico alguma vez na vida.

$\mathrm{Na}$ segunda etapa (etapa II), foram formados os dois grupos de estudo. Os escolares com asma foram alocados para o grupo de casos. Os controles foram pareados por sexo e idade, sendo que para cada aluno classificado com asma da escola, outros dois alunos, sem essa condição, da mesma escola e com a mesma idade e sexo, foram selecionados para participar do grupo controle. Foram excluídos do estudo escolares com limitações cognitivas e com outras doenças crônicas. A seleção dos participantes incluiu todos os casos identificados na primeira etapa e o número de controles necessário para perfazer dois controles para cada caso.

Ainda na etapa II, os responsáveis e os escolares selecionados para os dois grupos, em seus domicílios, responderam ao questionário de $\mathrm{QV}$ relacionada à saúde. Para esta etapa todos os participantes assinaram, além do TCLE, um termo de assentimento referente às atividades específicas dessa etapa (questionário de QV e medidas antropométricas). $\mathrm{O}$ instrumento utilizado para avaliação da QV foi o Kiddo-KINDL, previamente validado no Brasil para a população adolescente [13]. Esse questionário é composto de 24 itens divididos nos domínios bem-estar físico, bem-estar emocional, família, amigos e escola. Os valores atribuídos a cada resposta variam de 1 a 5 para as questões com direções positivas, e de 5 a 1 para as negativas. Os escores foram expressos pela média dos domínios, transformados em uma escala de 0 a 100, sendo que os valores mais próximos a 100 indicam melhor QV.

A avaliação do estado antropométrico foi realizada nas escolas, por pesquisadores treinados, utilizado o índice de massa corporal (IMC) relacionado com a idade em anos. Para o cálculo do IMC foi utilizado o Índice de Quetelet, obtido pela fórmula peso corporal $(\mathrm{kg})$ dividido pela estatura ao quadrado $\left(\mathrm{m}^{2}\right)$ [14]. As medidas do peso corporal e da estatura foram coletadas seguindo os critérios adotados pelo Sistema de Vigilância Alimentar e Nutricional do Ministério da Saúde [15].

Para a classificação do estado antropométrico pelo IMC, foram seguidos os critérios do Ministério da Saúde, utilizados nas Cadernetas de Saúde da Criança e do Adolescente, que preconizam como classificação do estado antropométrico o escore $\mathrm{Z}$, por entender que é a forma de mais fácil compreensão e utilização [16,17]. Para fins de análise estatística, os escolares classificados com sobrepeso e obesidade foram agrupados em um só grupo denominado sobrepeso/obesidade.
Para análise estatística dos dados, foi utilizado o programa IBM SPSS Statistics versão 23.0. As variáveis contínuas foram apresentadas como média e desviopadrão e as variáveis categóricas como frequência absoluta e relativa. As médias das pontuações obtidas em cada grupo foram comparadas utilizando o teste t de Student, considerando o nível de 5\% de significância.

\section{RESULTADOS}

$\mathrm{Na}$ etapa I do estudo foram investigados 1.691 estudantes, representando $84,5 \%$ de retorno dos questionários enviados. A média de idade dos respondentes foi de 11,55 $\pm 2,04$ anos, (7 a 18 anos), sendo 780 (46\%) do sexo masculino. De acordo com os critérios de classificação para asma, 184 escolares foram classificados com asma, representando uma prevalência de $10,8 \%$ na faixa etária estudada. Destes, dois estudantes foram excluídos do estudo por serem portadores de doença neurológica, 32 pais ou responsáveis não aceitaram participar do estudo, seis famílias mudaram de cidade e em 22 domicílios não foi possível realizar a entrevista por impossibilidade de agendamento, falha em contato telefônico ou por não se encontrarem em casa no dia e hora agendada, mesmo tendo sido feitas várias tentativas para agendamento. Dessa forma, 122 escolares com asma foram selecionados para a etapa II.

A seleção do grupo controle foi realizada partindo dos 184 casos encontrados, portanto 368 escolares sem asma foram convidados a participar do estudo, e 364 participaram. Os motivos das perdas foram semelhantes às dos casos, no entanto, quando os responsáveis negavam a participação, o escolar era substituído por outro com mesma idade e sexo que havia respondido à etapa $\mathrm{I}$.

Desta forma, no seguimento da etapa II, 486 escolares (122 casos e 364 controles) responderam ao questionário de QV. A avaliação do estado antropométrico foi realizada em 414 participantes (106 casos e 308 controles), constituindo a amostra final incluída no estudo. Dentre os motivos das perdas, incluem-se falta à aula no dia da coleta, recusa do escolar em realizar as avaliações e transferência para uma escola não participante do estudo.

Dentre os 414 escolares, 8 (1,9\%) foram classificados como baixo peso, $237(57,2 \%)$ como eutróficos, 90 (21,7\%) como sobrepeso e 79 (19,1\%) como obesos, portanto $169(40,8 \%)$ foram classificados com sobrepeso/obesidade. Sobrepeso/obesidade esteve presente em $49(46,2 \%)$ escolares do grupo asma e 
em 120 (38,9\%) dos controles, evidenciando maior proporção de sobrepeso/obesidade nos casos $(p<0,001)$. Os demais resultados quanto à caracterização da amostra participante da etapa II estão expressos na Tabela 1.

Comparando-se as médias dos escores de QV entre os grupos de casos e controles, observaram-se diferenças estatisticamente significativas no domínio bem-estar físico $(\mathrm{p}<0,001)$ e no escore total de QV ( $p=0,003)$, nos quais os escolares com asma apresentaram pior QV. Considerando toda a amostra, não foram encontradas diferenças estatisticamente significativas entre as médias dos escores de QV entre os escolares eutróficos e com sobrepeso/obesidade. Entretanto, considerando o grupo de escolares com asma e com sobrepeso/obesidade, a média do escore no domínio bem-estar físico apresentou-se menor em relação ao grupo de escolares sem asma e eutróficos $(\mathrm{p}=0,010)$. Estes valores estão expressos na Tabela 2.

\section{DISCUSSÃO}

O presente estudo evidenciou considerável prevalência de obesidade na amostra estudada, tanto para o grupo de escolares com asma, como para os controles. Os resultados referentes à $\mathrm{QV}$ entre os grupos mostram menores escores no domínio bem-estar físico e no escore total de QV para os escolares classificados com asma, e quando analisados estado antropométrico e QV, os escolares com asma e sobrepeso/obesidade apresentaram pior QV no domínio bem-estar físico quando comparados aos escolares controles e eutróficos.

O aumento da prevalência de obesidade e sobrepeso vem ocorrendo em todas as faixas etárias, tanto nos países desenvolvidos como em desenvolvimento [18]. No Brasil, o número de crianças acima do peso vem crescendo; de acordo com a Pesquisa de Orçamentos Familiares realizada pelo Instituto Brasileiro de Geografia e Estatística, houve um aumento do número

Tabela 1. Caracterização da amostra de escolares de terceiro a oitavo ano do ensino fundamental no município de ljuí/RS participantes da etapa II quanto a sexo, idade e estado antropométrico.

\begin{tabular}{|c|c|c|c|c|}
\hline Caraterística & $\begin{array}{l}\text { Grupo de casos (com asma) } \\
n=106\end{array}$ & $\begin{array}{l}\text { Grupo controle (sem asma) } \\
n=308\end{array}$ & $\mathbf{p}^{*}$ & $\begin{array}{c}\text { Total } \\
n=414\end{array}$ \\
\hline \multicolumn{5}{|l|}{ Sexo } \\
\hline Feminino $\mathrm{n}(\%)$ & $53(50)$ & $180(58,4)$ & 0,081 & $233(56,3)$ \\
\hline Masculino n (\%) & $53(50)$ & $128(41,6)$ & 0,141 & $181(43,7)$ \\
\hline \multicolumn{5}{|l|}{ Idade (anos) } \\
\hline Média \pm DP & $11,35 \pm 1,97$ & $11,62 \pm 2,06$ & 0,209 & $11,55 \pm 2,04$ \\
\hline \multicolumn{5}{|l|}{ IMC } \\
\hline Baixo peso ou eutrófico n (\%) & $57(53,8)$ & $188(61)$ & $<0,001$ & $245(59,1)$ \\
\hline Sobrepeso ou obesidade n (\%) & $49(46,2)$ & $120(39)$ & $<0,001$ & $169(40,8)$ \\
\hline
\end{tabular}

DP, desvio padrão; IMC, índice de massa corporal.

* Variáveis categóricas: teste Qui-quadrado. Variáveis contínuas: teste t de Student.

Tabela 2. Qualidade de vida entre os escolares de terceiro a oitavo ano do ensino fundamental no município de ljuí/RS, comparando o grupo com asma (casos) e o grupo sem asma (controles), os escolares com sobrepeso/obesidade e eutróficos nos dois grupos em conjunto, e a combinação das duas condições.

\begin{tabular}{|c|c|c|c|c|c|c|c|c|c|c|c|}
\hline $\begin{array}{c}\text { Domínios } \\
\text { da qualidade } \\
\text { de vida }\end{array}$ & sma & $\begin{array}{l}\text { Controles } \\
\text { (sem asma) }\end{array}$ & $\mathbf{p}$ & $\begin{array}{l}\text { Sobrepeso/ } \\
\text { obesidade }\end{array}$ & utróficos & p & $\begin{array}{c}\text { Asma + } \\
\text { sobrepeso/ } \\
\text { obesidade }\end{array}$ & $\begin{array}{c}\text { Sem asma }+ \\
\text { eutróficos }\end{array}$ & $\mathbf{p}$ & $\begin{array}{c}\text { Asma + } \\
\text { eutróficos* }\end{array}$ & $\mathbf{p}$ \\
\hline Bem-estar físico & $62,19 \pm 19,53$ & $72,25 \pm 16,67$ & $<0,001$ & $69,65 \pm 18,34$ & $70,60 \pm 17,25$ & 0,596 & $62,76 \pm 19,47$ & $70,47 \pm 17,64$ & 0,010 & $62,39 \pm 18,44$ & 0,921 \\
\hline Bem-estar emocional & $76,54 \pm 16,35$ & $79,45 \pm 15,80$ & 0,089 & $78,25 \pm 16,49$ & $79,84 \pm 15,60$ & 0,328 & $77,17 \pm 13,41$ & $78,88 \pm 16,25$ & 0,412 & $76,01 \pm 18,91$ & 0,718 \\
\hline Autoestima & $60,40 \pm 18,99$ & $59,76 \pm 20,21$ & 0,752 & $62,39 \pm 20,22$ & $58,52 \pm 19,29$ & 0,052 & $60,71 \pm 21,15$ & $59,84 \pm 19,76$ & 0,783 & $60,28 \pm 15,42$ & 0,903 \\
\hline Família & $71,67 \pm 17,10$ & $73,18 \pm 17,36$ & 0,403 & $72,74 \pm 17,28$ & $73,02 \pm 16,50$ & 0,871 & $70,03 \pm 18,26$ & $73,11 \pm 17,17$ & 0,264 & $73,55 \pm 16,56$ & 0,302 \\
\hline Amigos & $72,34 \pm 18,81$ & $75,44 \pm 16,24$ & 0,106 & $76,59 \pm 17,03$ & $74,23 \pm 16,33$ & 0,160 & $72,70 \pm 16,66$ & $74,87 \pm 17,01$ & 0,394 & $71,43 \pm 19,66$ & 0,724 \\
\hline Escola & $59,53 \pm 16,55$ & $61,81 \pm 16,25$ & 0,189 & $62,24 \pm 17,08$ & $60,21 \pm 15,43$ & 0,219 & $60,46 \pm 16,99$ & $61,32 \pm 16,28$ & 0,738 & $57,71 \pm 15,26$ & 0,384 \\
\hline Escore total & $67,11 \pm 10,41$ & $70,32 \pm 9,58$ & 0,003 & $70,31 \pm 10,19$ & $69,40 \pm 9,33$ & 0,357 & $67,30 \pm 9,90$ & $69,75 \pm 9,87$ & 0,107 & $66,89 \pm 10,19$ & 0,834 \\
\hline
\end{tabular}

Teste t de Student.

* Comparação com a coluna "Asma + sobrepeso/obesidade". 
de obesos em mais de $300 \%$ em crianças de cinco a nove anos, e na faixa etária de 10 a 19 anos aumentou em $20 \%$ [2].

No presente estudo, quando realizamos avaliação do estado antropométrico, encontramos prevalência de sobrepeso de $21,7 \%$ e de obesidade em $19,1 \%$. Rech et al. [18] avaliaram o estado antropométrico de 1.442 escolares de uma cidade serrana do Rio Grande do Sul e encontraram sobrepeso em $19,9 \%$, dado semelhante ao do presente estudo. Entretanto, a prevalência de obesidade foi menor naquele estudo, que identificou $8 \%$ de obesidade [18]. O estudo realizado por Costa et al. [19] na cidade de Santos, SP, em 10.822 escolares de 7 a 10 anos, encontrou prevalências menores: $15,7 \%$ dos escolares apresentaram sobrepeso e $18 \%$ obesidade.

Quando associados sobrepeso e obesidade nessa amostra, o grupo asma apresentou maior frequência dessa condição. No estudo realizado por Camilo et al. [20], 209 crianças e adolescentes hispânicos com e sem asma foram avaliados quanto ao estado antropométrico. No grupo de escolares com asma, $39,7 \%$ foram classificados com sobrepeso e $21,5 \%$ com obesidade, já no grupo de escolares sem a doença o percentual de sobrepeso foi de $32,9 \%$ e obesidade de $15,3 \%$ [20].

Alguns estudos recentes vêm levantando a hipótese de que a obesidade pode ser um fator de risco para a asma, e que a incidência de asma parece aumentar $50 \%$ em indivíduos com sobrepeso/obesidade $[21,22]$. A influência da obesidade nas doenças respiratórias pode causar uma desvantagem na mecânica pulmonar, diminuindo a capacidade funcional, volume pulmonar e o diâmetro das vias aéreas. $\mathrm{O}$ aumento de peso acaba impondo também uma resistência para a contração/ movimentação do diafragma, gerando uma carga inspiratória e restringindo as forças durante a insuflação/ desinsuflação dos pulmões, o que pode comprometer a mecânica muscular ventilatória [21,23]. Por outro lado, indivíduos com asma apresentam menor tolerância ao exercício físico devido às limitações causadas pela obstrução da via aérea no repouso e a ocorrência de broncoespasmo induzido pelo exercício. Essas alterações podem determinar a interrupção precoce da atividade física e um estilo de vida mais sedentário, aumentando o risco de sobrepeso/obesidade e reduzindo a $\mathrm{QV}$, especialmente no domínio bem-estar físico [20,24].

O impacto de doenças crônicas na QV dos indivíduos tem sido alvo de diversas pesquisas [8-10]. Neste estudo, quando comparamos a QV dos escolares entre os grupos asma e controle, encontramos diferenças significativas no domínio bem-estar físico e escore total, nos quais o grupo asma apresentou pior QV.

Quando consideramos os escolares divididos em grupo de sobrepeso/obesidade e eutróficos, o impacto destas variáveis na $\mathrm{QV}$ não foi significativo neste estudo. Na cidade de Porto Alegre, um estudo com 337 crianças e adolescentes encontrou resultados semelhantes, sem diferenças significativas na QV de acordo com as diferentes categorias do IMC [25]. Contudo, Poeta et al. [26], ao avaliarem 131 crianças obesas com idades entre oito e 12 anos, concluíram que essas crianças apresentavam QV inferior em todos os domínios em relação às crianças eutróficas.

Ao analisarmos a QV dos escolares com asma associada a sobrepeso/obesidade, encontramos menor escore no domínio bem-estar físico nesse grupo, quando comparados aos escolares sem as duas condições. Este resultado também foi evidenciado em um estudo com metodologia semelhante, onde 337 crianças e adolescentes foram avaliados e a QV foi reduzida na população com asma e sobrepeso/ obesidade em comparação a controles e eutróficos [25]. Além das alterações e limitações físicas causadas pela asma, o aumento de peso parece contribuir e aumentar as limitações nas atividades diárias de crianças e adolescentes [27], podendo influenciar de forma negativa na QV.

Uma das limitações deste estudo foi que apenas os escolares classificados com asma que aceitaram participar da etapa II responderam ao questionário de QV; todavia, o grupo controle foi pareado e selecionado de maneira aleatória, o que permite generalização dos dados. Outro fator de limitação foi a ausência de cálculo amostral, o que pode ter prejudicado a significância de alguns resultados. Também consideramos que houve muitas perdas entre as etapas I e II no grupo asma e entre aqueles que responderam ao questionário de QV e os que realizaram a avaliação antropométrica. Muitas famílias optaram em não participar do estudo, sendo essa a principal causa das perdas, seguida de muitos reagendamentos das entrevistas e ausência da família na residência nos horários previamente combinados, o que também demonstra o desinteresse em participar. A avaliação antropométrica foi realizada nas escolas, sem a presença dos responsáveis, o que de certa forma facilitou a recusa de alguns escolares em efetuar a avaliação.

As repercussões da asma na $\mathrm{QV}$ vêm sendo alvo de interesse. Considerando a variabilidade de fatores desencadeadores de crises de asma, as tentativas de se elucidar quais são os aspectos da doença e as comorbidades que interferem mais no dia a dia dos 
pacientes são importantes. A literatura científica que relaciona asma, obesidade e QV ainda é escassa. Neste sentido, há necessidade de mais pesquisas que esclareçam as relações entre essas condições, principalmente levando em conta o aumento da prevalência tanto da asma como da obesidade na população infantil. Este estudo mostrou que os escolares com asma apresentaram pior QV em relação aos controles sem asma, e quando considerados sobrepeso e obesidade como comorbidade da doença, a QV foi pior no domínio bem-estar físico.

\section{NOTA}

Declaração de conflitos de interesse

Os autores declaram não haver conflitos de interesse relevantes ao conteúdo deste estudo.

\section{REFERÊNCIAS}

1. Diretrizes da Sociedade Brasileira de Pneumologia e Tisiologia para o manejo da asma. J Bras Pneumol. 2012;38 Supl 1:S1-46.

2. Andrade LS, Araújo AC, Cauduro TM, Watanabe LA, Castro AP, Jacob CM, Pastorino AC. Obesidade e asma: associação ou epifenômeno? Rev Paul Pediatr. 2013;31(2):138-44. http://dx.doi.org/10.1590/S0103-05822013000200002

3. Global Initiative for Asthma. Global strategy for asthma management and prevention [Internet]. 2016 [cited 2011]. Available from: www. ginasthma.org

4. Schachter LM, Peat JK, Salome CM. Asthma and atopy in overweight children. Thorax. 2003;58:1031-5. http://dx.doi.org/10.1136/ thorax.58.12.1031

5. Von Mutius E, Schwartz J, Neas LM, Dockery D, Weiss ST. Relation of body mass index to asthma and atopy in children: the National Health and Nutrition Examination Study III. Thorax. 2001;56(11):835-8. http://dx.doi.org/10.1136/thorax.56.11.835

6. Pianosi PT, Davis HS. Determinants of physical fitness in children with asthma. Pediatrics. 2004;113(3 Pt 1):e225-9. http://dx.doi. org/10.1542/peds.113.3.e225

7. Lucas SR, Platts-Mills TA. Pediatric asthma and obesity. Paediatr Respir Rev. 2006;7(4):233-8. http://dx.doi.org/10.1016/j.prrv.2006.08.001

8. Nogueira KT, Silva JRL, Lopes CS. Qualidade de vida em adolescentes asmáticos: avaliação da gravidade da asma, comorbidade e estilo de vida. J. Pediatr. 2009;85(6):523-30. http://dx.doi.org/10.1590/s0021-75572009000600009

9. Velsor-Friedrich BV, Militello LK, Kouba J, Harrison PR, Manion A, Doumit R. Pediatric Obesity and Asthma Quality of Life. Nursing Clinics of North America. 2013;48(2):259-70. http://dx.doi.org/10.1016/j.cnur.2013.01.011

10. Gent RV, Ent CV, Rovers MM, Kimpen JL, Essen-Zandvliet LE, de Meer G. Excessive body weight is associated with additional loss of quality of life in children with asthma. J Allergy Clin Immunol. 2007;119(3):591-6. http://dx.doi.org/10.1016/j.jaci.2006.11.007

11. Cassol VE, Rizzato TM, Teche SP, Basso DF, Centenaro DF, Maldonado M, Moraes EZ, Hirakata VN, Solé D, Menna Barreto SS. Obesity and its relationship with asthma prevalence and severity in adolescents from southern Brazil. J Asthma. 2006;43:57-60. http://dx. doi.org/10.1080/02770900500448597

12. Sole D, Yamada E, Vana AT, Werneck G, Solano de Freitas L, Sologuren MJ, Brito M, Rosário Filho NA, Stein RT, Mallol J. International Study of Asthma and Allergies in Childhood (ISAAC): prevalence of asthma and asthma-elated symptom samong Brazilian school children. J Investig Allergol Clin Immunol. 2001;11(2):123-8.

13. Teixeira IP Novais IP, Pinto RMC, Cheik NC. Cultural adaptation and validation of the KINDL questionnaire in Brazil for adolescents between 12 and 16 years of age. Rev Bras Epidemiol. 2012;15(4):845-57. http://dx.doi.org/10.1590/S1415-790X2012000400015

14. Vitolo MR. Avaliação nutricional da criança. In: Nutrição: da gestação ao envelhecimento. 2th ed. Rio de Janeiro: Ed. Rubio; 2014.

15. Ministério da Saúde (BR). Secretaria de Atenção à Saúde. Departamento de Atenção Básica. Orientações para a coleta e análise de dados antropométricos em serviços de saúde [Internet]: Norma Técnica do Sistema de Vigilância Alimentar e Nutricional. Brasília: Ministério da Saúde; 2011 [cited 2016]. Available from: http://dab.saude.gov.br

16. Ministério da Saúde (BR). Secretaria de Atenção à Saúde. Departamento de Ações Programáticas Estratégicas. Caderneta de Saúde da Criança [Internet]. Passaporte da Cidadania. 6a ed. Brasília: Ministério da Saúde; 2009 [cited 2016]. Available from: http://bvsms. saude.gov.br

17. Ministério da Saúde (BR). Secretaria de Atenção à Saúde. Departamento de Ações Programáticas Estratégicas. Caderneta de Saúde do Adolescente [Internet]. Brasília: Ministério da Saúde; 2009 [cited 2016]. Available from: http://bvsms.saude.gov.br

18. Rech RR, Halpern R, Costanzi CB, Bergmann MLA, Alli LR, Mattos AP, Trentin L, Brum LR. Prevalência de obesidade em escolares de 7 a 12 anos de uma cidade Serrana do RS, Brasil. Rev Bras Cineantropom Desempenho Hum. 2010;12(2):90-7.

19. Costa RF, Cintra IP, Fisberg M. Prevalência de sobrepeso e obesidade em escolares da cidade de Santos, SP. Arq Bras Endocrinol Metab. 2006;50(1):60-7. http://dx.doi.org/10.1590/S0004-27302006000100009

20. Camilo DF, Ribeiro JD, Toro AD, Baracat EC, Barros Filho AA. Obesity and asthma: association or coincidence? J Pediatr. 2010;86(1): 6-14. http://dx.doi.org/10.2223/jped.1963 
21. Brashier B, Salvi S. Obesity and asthma: physiological perspective. J Allergy (Cairo). 2013;2013:198068. http://dx.doi. org/10.1155/2013/198068

22. Sin DD, Sutherland ER. Obesity and the lung: 4 .Obesity and asthma. Thorax. 2008;63(11):1018-23. http://dx.doi.org/10.1136/ thx.2007.086819

23. Lucas SR, Platts-Mills TA. Paediatric asthma and obesity. Paediatr Respir Rev. 2006;7(4):233-8. http://dx.doi.org/10.1016/j.prrv.2006.08.001

24. Rubin AS, Pereira CAC, Neder JA, Fitermsn J, Pizzichini MMM. Hiperresponsividade brônquica. J Pneumol. 2002;28(Supl 3):101-21.

25. Filho JPH. Impacto da asma sobre qualidade de vida, sedentarismo e capacidade muscular ventilatória e a influência do sobrepeso/obesidade em crianças e adolescentes [dissertação]. Porto Alegre: PUCRS; 2014.

26. Poeta LS, Duarte MFS, Giuliano ICB. Qualidade de vida relacionada à saúde de crianças obesas. Rev Assoc Med Bras. 2010;56(2): 168-72. http://dx.doi.org/10.1590/S0104-42302010000200014

27. Priftis KN, Panagiotakos DB, Anthracopoulos MB, Papadimitriou A, Nicolaidou P. Aims, methods and preliminary findings of the Physical Activity, Nutrition and Allergies in Children Examined in Athens (PANACEA) epidemiological study. BMC Public Health. 2007 July 4;7:140. http://dx.doi.org/10.1186/1471-2458-7-140 